\title{
Bioanalysis
}

\section{Applied Pharmaceutical Analysis India 2014 conference report}

\author{
Applied Pharmaceutical Analysis (APA) India
}

\section{3-26 February 2014, Ahmedabad, India}

The fifth Applied Pharmaceutical Analysis (APA) India meeting was held in February 2014 at Hyatt Ahmedabad, India. With the theme of 'The Science of Measurement: Current status and Future trends in Bioanalysis, Biotransformation and Drug Discovery Platforms', the conference was attended by over 160 delegates. The agenda comprised advanced and relevant research topics in the key areas of bioanalysis and drug metabolism. APA India 2014 provided a unique platform for networking and professional linking to participants, innovators and policy-makers. As part of the global research community, APA India continues to grow and receive considerable attention from the drug discovery and development community of India.

The Applied Pharmaceutical Analysis (APA) India 2014 meeting, an initiative by the nonprofit organization The Boston Society, Inc. (MA, USA) is a continued effort towards providing a unique platform for drug discovery and development scientists for knowledge sharing through the organization of short courses and workshops in the areas of discovery bioanalysis, biotransformation and regulated bioanalysis [1]. Translational in its nature, this annual meeting receives participation from both academia and industry sectors from across the globe. The fifth iteration of this conference, with the them 'The Science of Measurement: Current Status and Future Trends in Bioanalysis, Biotransformation and Drug Discovery Platforms', was held from 23-26 February 2014 at Hyatt Ahmedabad, India. The event was structured as 1 day of short courses (23 February), followed by 3 days of dedicated workshops on regulated bioanalysis (24 February), biotransformation (25 February) and discovery bioanalysis (26 February). Following the success of the past years, the 2014 meeting received overwhelming participation from over 160 delegates representing drug discovery, contract research organizations, generic manufacturers, biosimilars/biologics sections of industry and research-driven governmen- tal and nongovernmental academic institutes. This article is an attempt to summarize the key highlights of this meeting and the key topics/discussions for the broader drug discovery and development community.

\section{APA India 2014 short courses}

As is traditional, APA 2014 started with short courses that were designed to provide delegates with the latest technical developments in the areas of biotransformation and bioanalysis. The short courses provide an opportunity for the participants to obtain hands-on experience with various analytical techniques or learn through a variety of case studies, giving greater perspective than the literature.

\section{Biotransformation short course}

The day began with a talk by Punit Marathe (BMS, NY, USA) entitled 'Pharmacokinetics (PK) in Pharmaceutical Discovery and Development', which covered PK studies in preclinical species, the relevance of key PK parameters, the utility of toxicokinetic studies and the prediction of human PK parameters from preclinical studies through allometric scaling and other methodologies. Case studies such as the specific cases of drug candidates differentially binding to $\alpha 1$-acid
Prashant Kole*,1, Deepak Barot $^{2}$, Jignesh Kotecha ${ }^{3}$, Vijay Raina ${ }^{4}$, Mukkavilli Rao ${ }^{5}$ \& Manish Yadav 6

'Pharmaceutical Candidate Optimization, Biocon Bristol-Myers Squibb R\&D Centre (BBRC), Syngene International Ltd, Biocon Park, Plot 2 \& 3, Bommasandra IV Phase, Bangalore 560 099, Karnataka, India

${ }^{2}$ Preclinical Research, 283, Sector 27, Gayatri Society, Gandhinagar 382028, Gujarat, India

${ }^{3}$ Torrent Pharmaceuticals Ltd, Research Centre, PO Bhat, Gandhinagar 382428, Gujarat, India

${ }^{4}$ Nektar Therapeutics (India) Private Ltd, Genome Valley, Lalgadi Malakpet, Shamirpet RR District, Hyderabad 500078, Andhra Pradesh, India ${ }^{5}$ Advinus Therapeutics Ltd, Plot No. 21, 22, Phase 2, Peenya Industrial Area, Bangalore 560058, Karnataka, India ${ }^{6}$ Alkem Labs, India, B-23, Krishna Bunglows, Nr. Basant Bahar 1, Gala Gymkhana Road, Ahmedabad 380058, Gujrat, India

*Author for correspondence:

Tel.: +919945042200

Fax: +91 8028084023

prashant.kole@syngeneintl.com 
glycoprotein in human and preclinical species (rat) and non-linear PK profiles relating to solubility/dissolution rate limited absorption were key highlights of the presentation. This talk was attended by the attendees of both the biotransformation and bioanalysis short courses. Subrahmanyam Vangala (Advinus, India) delivered the second lecture entitled 'Hepatotoxicity: Mechanisms, Models and Predictions', presenting case studies relating to hepatotoxicity, which is the major reason for postmarketing drug withdrawal, alongside various mechanisms that could cause hepatotoxicity and the possible reasons for the poor translation of animal model studies to humans. Kaushik Mitra (Merck, NY, USA) gave a talk titled 'Metabolism in Drug Candidates'. Mitra was of the opinion that early de-risking of lead compounds is more prudent than late-stage surprises in drug development. He advocated integration of reactive metabolite studies right at the drug design stage and for studies to be carried out in early structure-activity relationships (SARs) development. He emphasized structure-based reactive metabolite liabilities (potential binding to DNA and proteins) and also briefed on various trapping strategies.

The following sessions focused on drug transporters and the talks were delivered by William Elmquist (University of Minnesota, MN, USA) and Jasminder Sahi (GSK, China). Elmquist gave a talk entitled 'Transporters in ADME - Targeted Bioavailability' and discussed the colocalization of transporters with drugmetabolizing enzymes, relating it to the variability in drug absorption and disposition [2]. He specifically discussed the example of sorafenib [3], which is a substrate of both P-gp and BCRP, and how the knockout of both transporters at the blood-brain barrier acted synergistically, leading to increases in brain exposure in comparison with single transporter-knockout mice. The day ended with a presentation by Sahi entitled 'Species Differences in Drug Transporters: Implications in Safety and Efficacy'. She used various examples to show the differences in ATP Binding Cassette (ABC) and solute carrier (SLC) transporters between preclinical species and humans at the blood-brain barrier, liver and kidney. She opined on the significance of a better understanding of species differences in transporter expression for a better mechanistic understanding of drug dispositions and to further build translational PK/pharmacodynamics or toxicology models accounting for these species differences.

\section{Bioanalysis short course}

The bioanalysis short course started with a brief talk by Deepak Barot (Preclinical Research, India), who set the tone by highlighting the key roles and responsibilities of the discovery bioanalyst. The session continued with a talk delivered by Prashant Kole (BBRC, India) on the development and validation of dried blood spot (DBS) assays. Kole detailed how dried matrices are different from wet matrices and, through case studies, explained the importance of sample preparation strategies, specifically for clinical pediatric/neonatal samples [4]. Rajan Kombu (Aizant, India) delivered the next talk entitled 'Metabolomics Using Mass Spectrometer'. He presented case studies advocating the use of isotopomer analysis [5] to study metabolism, biological energy flux, biological synthesis and pathway identification, turnover of metabolites and rate of synthesis of polymeric molecules. The next talk of the session was delivered by Manish Yadav (Alkem, India) in the area of regulated bioanalysis and provided current updates with respect to the characteristics of bioanalytical methods, current practices in bioanalytical method validation (BMV) and regulated bioanalysis, global best practices and the harmonization of bioanalytical guidance.

Post-lunch sessions were conducted at the Thermo Fisher Center of Excellence for Chromatography Consumables (Thermo Fisher, Ahmedabad, India), which included hands-on training sessions. Tushar Mehta (Thermo Fisher, India) introduced participants to the objectives and functions of the center. Rajaram Patil and Krishna Dara (Thermo Fisher, India) delivered talks on liquid chromatography-tandem mass spectrometry platforms for bioanalysis and recent trends in sample preparation, respectively. The talks were followed by a laboratory visit and a demonstration of new sample preparation technologies. The laboratory session was highly interactive and participants shared their views and experiences on different sample preparation products.

\section{Regulated bioanalysis workshop: day 1 (24 February 2014)}

The first dedicated day session of APA 2014 for regulatory bioanalysis started with a welcome note from Sandhya Mandlekar (BBRC, India), followed by an introduction of the conference topics by Deepak Barot.

The plenary session comprising two talks was opened by Vijay Raina (Nektar Therapeutics, India), with an emphasis on enriching knowledge and the value that the APA Society brings to participants. Along with Manish Yadav, Raina moderated the inaugural talks. The first keynote address on regulatory bioanalysis was delivered by Kiran Marthak (Lambda Therapeutics, India). Marthak presented the current regulatory scenario for clinical research in India. He provided insight into recent changes to regulatory policies, such as the introduction of new policies, rules and regulations for the conductance of clinical trials in India and 
its business impact on the Indian contract research organization (CRO) industry. Marthak informed us that concerns are being brought to the government's attention through various forums, such as the Association of Contract Research Organizations (ACRO) and the Organisation of Pharmaceutical Producers of India (OPPI), and emphasized that representatives of these forums are working closely with regulatory agencies in order to streamline the process through the continuous engagement and training of regulatory staff. The second plenary talk was delivered by Bhaswat Chakraborty (Cadila Pharma, India), highlighting the analysis and approval of biosimilars through case studies and data. Chakraborty emphasized structural analysis (including aggregation and post-translation modification), in addition to protein and immunogenicity determinations of biosimilar drug products.

The first technical session of the day was dedicated to regulated bioanalysis methods for large molecules and biomarkers. The first talk of the session was delivered by Anita Krishnan (Lupin, India), who emphasized future challenges in peptide and protein analysis. Krishnan also discussed two tools for studying higher-order protein structure, namely second-derivative ultraviolet and hydrogen-deuterium exchange. The next talk was delivered by Kakali Dhar (Biocon, India), who presented the challenges of biologics with a focus on method validation and sample analysis using ligand-binding assays and described the similarities and differences in bioanalytical guidelines. Deepa Joshi (BBRC, India) presented the development and validation of a fit-for-purpose biomarker assay and discussed how this validation balances the burden and stringency of proof required in order to validate an assay with regards to the technology and applicability of biomarker. Kiernan Urbana (Thermo Scientific, AZ, USA) delivered a talk on mass spectrometric immunoassays in insulin analytics, which is a high-throughput assay for the quantification of insulin and its analogs from human plasma samples.

The second session was dedicated to method development, validation and sample analysis, which began with a talk by Ravi Shankar (GVK Bio, India) addressing incurred sample stability and regulatory perspectives. He presented different scenarios of incurred sample stability in order to address incurred sample reanalysis (ISR) failure and discussed the need for this at various stages of bioanalysis. In the second talk, Aslam Khan (Reliance Life Sciences, India) emphasized the importance of the quality of reference standards, the impurities that may be present and their impact on the accuracy of analytical results through case studies.

Session three began with a talk from Manish Yadav, who covered the current challenges with respect to the quality, safety and efficiency management of modern bioanalytical laboratories. He also touched upon the latest practices for handling patient samples. Yadav further emphasized that successful laboratory management and patient sample handling not only requires sound scientific, quality and regulatory knowledge, but also demands awareness of safety measures. In the second talk, Neerja Somyajee (Piramal, India) spoke about practices and procedures that enabled successful handling of regulatory inspections. The session ended with Mark Arnold's (BMS, NY, USA) webinar entitled 'Regulatory Guidelines - FDA, EMEA, ANVISA: Harmonization, Guidelines and Updates'. He provided a perspective of industry and the US FDA during the Crystal City V conference (3-5 December 2013, Baltimore, MD, USA). Arnold's updates were able to provide the audience with the current thinking of the FDA and global bioanalytical leaders on longstanding and puzzling issues of regulated bioanalysis. Finally, Puran Singhal (Alkem, India) shared the latest updates on Global Bioanalysis Consortium (GBC) activities.

\section{Biotransformation workshop: day 2 (25 February 2014)}

The second day of the APA 2014 meeting was focused on the principles of biotransformation and drug transporters in understanding the ADME (absorption, distribution, metabolism and excretion) properties of new chemical entities. The keynote address was delivered by William Elmquist, who elaborated on the role of drug transporters at the blood-brain barrier (BBB) and their impact on the efficacy of molecularly targeted agents. In the first session, Dhiren Thakker (UNC Eshelman School of Pharmacy, NC, USA) talked about role of intestinal transporters in the transport of cationic, anionic and zwitterionic drugs. The next talk by Jasminder Sahi discussed how tight junctions and efflux transporters at the BBB could limit the penetration of drugs into the CNS. She provided interesting case studies on how targeting uptake transporters such as OATP1A2 at the BBB could overcome the barrier and enhance the distribution of drugs into the brain.

The second session of the day was focused on understanding the intricacies of the biotransformation pathways and various strategies for elucidating them. The first talk in this session was delivered by Kaushik Mitra, entitled 'Deciphering of Unusual Biotransformations in Real-Time Strategy and Impact on Drug Discovery'. Mitra exemplified his point by showing case studies from his experience and the various strategies he has adopted to interpret and decipher the biotransformation pathways and hence developing a good SAR for lead optimization. The subsequent 
talk was by Subrahmanyam Vangala on the use of the coadministration of $\beta$-glucuronidase as a strategy for understanding the disposition of compounds that are extensively glucuronidated and undergo enterohepatic recirculation. Vangala presented an example from his previous research as to how glucuronide conjugates are excreted through bile, hydrolyzed to the parent by $\beta$-glucuronidase in the intestine and reabsorbed into systemic circulation. The last talk in this session was by Syed Lateef (Agilent Technologies, India) on 2D liquid chromatography-tandem mass spectrometry technology. Agilent Technologies developed a method of using binary pumps and a trapping and analytical column in order to elute analyte(s) and metabolite(s). The panel discussion following these talks extensively focused on issues related to the early de-risking of molecules and balancing risk versus benefit.

Session three featured a talk by Michael Sinz (BMS, NY, USA) on the effect of a single-nucleotide polymorphism of the coxsackie and adenovirus receptor (CAR) promoter on enzyme induction. Sinz mentioned the prevalence of single-nucleotide polymorphisms and how these variants could impact on the extent of CYP induction. The subsequent talk was by Murali Subramanian (BBRC, India) on the transfection of cynomolgus monkey CYP3A4 and CYP3A5 [6]. Subramanian presented data on the expression and characterization of monkey CYP3A4 and CYP3A5 in appropriate vectors and their utility in drug-drug interaction (DDI) screening in early discovery. The last talk in this session was by Michael Bolger (Simulations Plus, CA, USA). Bolger explained the interpretation of unusual PK behavior and provided some strategies for predicting PK in humans using physiologically based PK modeling.

The last session of the day comprised talks on the PK and pharmacodynamics of biologics and the use of accelerated mass spectrometry in the metabolite identification of small molecules early in drug development. Gabriel Robbie (Medimmune, DC, USA) gave insights into biologic therapies. Robbie presented the key differences in the characteristics of biologics and small molecules, their PK/pharmacodynamic behavior and the development of relevant assays and bioanalytical methods for monitoring them. Robbie also dealt with the regulatory perspective and the selection of safe starting doses in humans for biologics. Swamy Yeleswaram (Incyte, PA, USA) spoke about the current FDA and International Conference on Harmonisation guidelines and how important it is to identify the major human circulating metabolites at an early stage, rather waiting until the publication of large-scale efficacy trials. He presented an alternative strategy of using a microtrace amount of ${ }^{14} \mathrm{C}$-labeled compounds along with cold compounds during Phase I clinical studies and the utilization of accelerated mass spectrometry in order to establish a quantitative assessment of major human metabolites.

\section{Discovery bioanalysis workshop: day 3 (26 February 2014)}

The last day was focused on discovery bioanalysis, which was categorized into three sessions; drug discovery-preclinical phase; large-molecule bioanalysis; and sample preparation and newer technologies. The day started with the first keynote presentation by Anjani Kumar (National Institute of Animal Welfare, Government of India), who provided insight into animal ethics and welfare in India, covering the related rules, regulations and policies. He explained the current guidelines and changes in the regulations for the care, maintenance and use of laboratory animals in preclinical drug discovery. He emphasized the principles of replacement, reduction and refinement (known as the 3Rs) in animal experiments. The second keynote speaker was Jyoti Paliwal (Troikka Pharma, India), who shared his thoughts on drug discovery scenarios in India and shared some success stories of Indian pharmaceutical companies. Paliwal insisted on the benefits of the collaborative research model between academia and industry for obtaining more success stories in the future.

The first talk of the 'drug discovery-preclinical phase' session was delivered by Sandhya Mandlekar, who explained the utility of animal models for predicting CYP3A4 induction/inhibition and hepatic uptake transporter-mediated drug-drug interactions. Mandlekar explained the limitations of in vitro tools for predicting the DDI potential and rationale for the development of specific animal models for the prediction of human DDIs for new chemical entities, along with their caveats. The second talk of the session was delivered by Punit Marathe. Marathe's talk was focused on low-clearance compounds and the challenges in in vitro, in vivo and in vitro-in vivo extrapolation studies. She illustrated newer techniques for estimating the clearance of low-turnover compounds, presented case studies where in vivo clearance could not be predicted from in vitro tools and discussed alternative strategies that might help mitigate the risk in predicting human PK profiles.

The large-molecule bioanalysis session started with a talk by Himanshu Gadgil (Intas Pharma, India), who elaborated on the role of differentiated analytics in the analysis of biologics/large molecules. Gadgil explained the stability and post-translational changes/challenges in the characterization of biologics. He detailed various analytical tools, the use of intrinsic internal standards 
for the liquid chormatography-mass spectrometry estimation of large molecules. Yatin Gokarn (Institute of Chemical Technology, India) presented a second talk focusing on the need for, logic of and approach to characterizing active biomolecules and other drug product components (i.e., excipients), including their potential degradation and interactions. Gokarn also touched upon various characterization tools for identifying the potential changes to protein molecules during formulation, packaging and drug delivery [7]. The third talk of the session was delivered by Sudarshan Mantha (Waters, India), who discussed the key challenges (e.g., molecular nature, specificity, sensitivity, carry-over and nonspecific binding, among others) in peptide bioanalysis and suggested strategies to overcome these challenges with various case studies.

The third session of the day was dedicated to newer sample collection/preparation techniques and related technologies. Vishwottam Kandikere (Advinus, India) started the session with a talk on plasma microsampling techniques for reducing the sampling volume in preclinical $\mathrm{PK} /$ toxicokinetic studies. He also elaborated on dialysis and capillary microsampling techniques [8-10] with relevant case studies. Echoing the microsampling theme, the second talk was delivered by Vijay Raina, who touched upon the DBS technique, highlighting the importance of sequential method development strategies in order to establish a sensitive and reproducible bioanalytical method. The next talk on this theme was delivered by Prashant Kole, who detailed the conceptual shift in bioanalytical sample matrices from 'wet' to 'dry'. He touched upon various dried matrices and specifically discussed the transformation of the dried blood 'technique' to technology. He gave an account of various instrumentation platforms of DBS technology and updated the audience with recent developments in the perceptions of regulatory agencies regarding the DBS methodology. The last talk of the session was delivered by Vijayabhaskar Veeravalli (GVK, India), detailing the effects of common discovery formulation excipients on bioanalysis and discussing approaches to identifying and nullifying the matrix effects caused by them [11,12]. The last session of the day comprised three webinars. Jonathan Josephs (BMS, NJ, USA) discussed assay development and data processing using two high-resolution mass spectrometry instrumentation platforms (i.e., time of flight and orbitrap). Stacy Ho (Sanofi, MA, USA) examined surrogate matrices for tissue bioanalysis. She discussed the advantages and disadvantages of the use of surrogate matrices and suggested considerations for the selection of appropriate surrogate biomatrices for a particular bioanalysis. She also discussed a case study comprising the use of a surrogate matrix in the esti- mation of endogenous compounds in tissue samples. A webinar on studies with radiolabeled compounds was presented by Ramaswamy Iyer (BMS, NJ, USA).

Interactive panel discussions were held after each of the sessions on all 3 days of the workshop and were highly appreciated, as they a unique opportunity to both the speakers and delegates to discuss various topics of relevance and importance. The APA 2014 meeting ended with thanks being given to all of the organizers, sponsors, speakers and delegates. The entire event was well received by the participants (speakers and delegates) and all appreciated the success of the event.

\section{Summary}

The fifth iteration of the APA meeting again made a mark for the drug discovery and development scientific community in India. The theme, agenda and global key speakers of the event attracted over 160 delegates from various sectors of Indian drug discovery and development industry/academia. For the first time, the APA meeting arranged on-site, hands-on training opportunities for the bioanalysis short course attendees, who gave very positive feedback and received insights into various recent technologies and instrument platforms. Growing interest in the area of biotransformation across the drug discovery and CRO industry was evident, and attendees benefited from the varied topics and the discussion of cutting-edge methodologies. Topics such as microsampling and microanalysis continue to attract regulated and nonregulated bioanalytical researchers and is gaining ground in the Indian drug development industry.

The APA 2014 meeting provided a vital opportunity for participants to learn, discuss, exchange views and network with the local and global research community at a single forum. The meeting is increasingly being considered as a premier conference in India for researchers in the pharmaceutical drug discovery and development industry.

\section{Acknowledgements}

The authors acknowledge M Subramanian (BBRC, India) for his help in preparing this manuscript.

\section{Financial \& competing interests' disclosure}

The authors have no relevant affiliations or financial involvement with any organization or entity with a financial interest in or financial conflict with the subject matter or materials discussed in the manuscript. This includes employment, consultancies, honoraria, stock ownership or options, expert testimony, grants or patents received or pending, or royalties.

No writing assistance was utilized in the production of this manuscript. 


\section{References}

1 Kole P, Shedbalkar V, Raina V et al. Applied Pharmaceutical Analysis India 2013 conference report. Bioanalysis 5(15), 1821-1825 (2013).

2 Agarwal S, Manchanda P, Vogelbaum MA et al. Function of the blood-brain barrier and restriction of drug delivery to invasive glioma cells: findings in an orthotopic rat xenograft model of glioma. Drug Metab. Dispos. 41(1), 33-39 (2013).

3 Oberoi RK, Mittapalli RK, Elmquist WF. Pharmacokinetic assessment of efflux transport in sunitinib distribution to the brain. J. Pharmacol. Exp. Ther. 347(3), 755-764 (2013).

4 Suyagh MF, Kole PL, Millership J et al. Development and validation of a dried blood spot-LC-APCI-MS assay for estimation of canrenone in paediatric samples. J. Chromatogr. B Analyt. Technol. Biomed. Life Sci. 878(9-10), 769-776 (2010).

5 Yang L, Kombu RS, Kasumov T et al. Metabolomic and mass isotopomer analysis of liver gluconeogenesis and citric acid cycle. I. Interrelation between gluconeogenesis and cataplerosis; formation of methoxamates from aminooxyacetate and ketoacids. J. Biol. Chem. 283(32), 21978-21987 (2008).

6 Selvakumar S, Bhutani P, Ghosh K et al. Expression and characterization of cynomolgus monkey cytochrome CYP3A4 in a novel human embryonic kidney cell-based mammalian system. Drug Metab. Dispos. 42(3), 369-376 (2014).

7 Brych SR, Gokarn YR, Hultgen $\mathrm{H}$ et al. Characterization of antibody aggregation: role of buried, unpaired cysteines in particle formation. J. Pharm. Sci. 99(2), 764-781 (2010).

8 Jonsson O, Palma Villar R, Nilsson LB et al. Capillary microsampling of $25 \mu \mathrm{l}$ blood for the determination of toxicokinetic parameters in regulatory studies in animals. Bioanalysis 4(6), 661-674 (2012).

9 Jonsson O, Villar RP, Nilsson LB et al. Validation of a bioanalytical method using capillary microsampling of $8 \mu \mathrm{l}$ plasma samples: application to a toxicokinetic study in mice. Bioanalysis 4(16), 1989-1998 (2012).

10 Jonsson O, Steffen AC, Sundquist VS et al. Capillary microsampling and analysis of $4-\mu \mathrm{l}$ blood, plasma and serum samples to determine human $\alpha$-synuclein elimination rate in mice. Bioanalysis 5(4), 449-462 (2013).

11 Vijaya Bhaskar V, Middha A. Liquid chromatography/ tandem mass spectrometry method for quantitation of Cremophor EL and its applications. Int. J. Anal. Chem. 135613 (2013).

12 Vijaya Bhaskar V, Middha A, Tiwari S et al. Liquid chromatography/tandem mass spectrometry method for quantitative estimation of polyethylene glycol 400 and its applications. J. Chromatogr. B Analyt. Technol. Biomed. Life Sci. 926, 68-76 (2013). 